NASA/TM-2003-212657

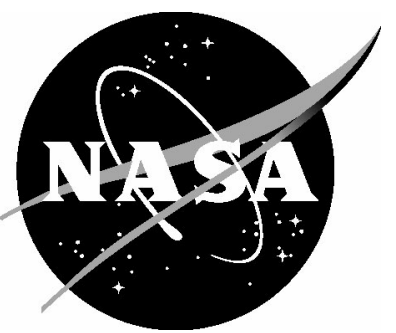

Evaluation of a Singular Value Decomposition Approach for Impact Dynamic Data Correlation

Lucas G. Horta, Karen H. Lyle, and Wendy B. Lessard

Langley Research Center, Hampton, Virginia 
Since its founding, NASA has been dedicated to the advancement of aeronautics and space science. The NASA Scientific and Technical Information (STI) Program Office plays a key part in helping NASA maintain this important role.

The NASA STI Program Office is operated by Langley Research Center, the lead center for NASA's scientific and technical information. The NASA STI Program Office provides access to the NASA STI Database, the largest collection of aeronautical and space science STI in the world. The Program Office is also NASA's institutional mechanism for disseminating the results of its research and development activities. These results are published by NASA in the NASA STI Report Series, which includes the following report types:

- TECHNICAL PUBLICATION. Reports of completed research or a major significant phase of research that present the results of NASA programs and include extensive data or theoretical analysis. Includes compilations of significant scientific and technical data and information deemed to be of continuing reference value. NASA counterpart of peerreviewed formal professional papers, but having less stringent limitations on manuscript length and extent of graphic presentations.

- TECHNICAL MEMORANDUM. Scientific and technical findings that are preliminary or of specialized interest, e.g., quick release reports, working papers, and bibliographies that contain minimal annotation. Does not contain extensive analysis.

- CONTRACTOR REPORT. Scientific and technical findings by NASA-sponsored contractors and grantees.
- CONFERENCE PUBLICATION. Collected papers from scientific and technical conferences, symposia, seminars, or other meetings sponsored or co-sponsored by NASA.

- SPECIAL PUBLICATION. Scientific, technical, or historical information from NASA programs, projects, and missions, often concerned with subjects having substantial public interest.

- TECHNICAL TRANSLATION. Englishlanguage translations of foreign scientific and technical material pertinent to NASA's mission.

Specialized services that complement the STI Program Office's diverse offerings include creating custom thesauri, building customized databases, organizing and publishing research results ... even providing videos.

For more information about the NASA STI Program Office, see the following:

- Access the NASA STI Program Home Page at http://www.sti.nasa.gov

- E-mail your question via the Internet to help@sti.nasa.gov

- Fax your question to the NASA STI Help Desk at (301) 621-0134

- Phone the NASA STI Help Desk at (301) 621-0390

- Write to:

NASA STI Help Desk

NASA Center for AeroSpace Information

7121 Standard Drive

Hanover, MD 21076-1320 
NASA/TM-2003-212657

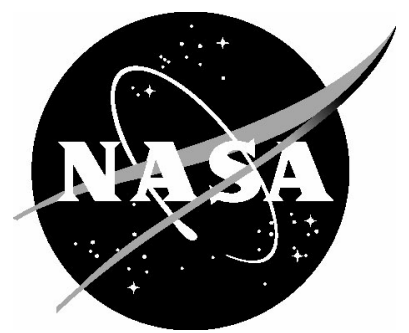

\section{Evaluation of a Singular Value Decomposition Approach for Impact Dynamic Data Correlation}

Lucas G. Horta, Karen H. Lyle, and Wendy B. Lessard

Langley Research Center, Hampton, Virginia

National Aeronautics and

Space Administration

Langley Research Center

Hampton, Virginia 23681-2199

October 2003 
Available from:

NASA Center for AeroSpace Information (CASI)

7121 Standard Drive

Hanover, MD 21076-1320

(301) 621-0390
National Technical Information Service (NTIS) 5285 Port Royal Road Springfield, VA 22161-2171

(703) 605-6000 


\begin{abstract}
Impact dynamic tests are used in the automobile and aircraft industries to assess survivability of occupants during crash, to assert adequacy of the design, and to gain federal certification. Although there is no substitute for experimental tests, analytical models are often developed and used to study alternate test conditions, to conduct trade-off studies, and to improve designs. To validate results from analytical predictions, test and analysis results must be compared to determine the model adequacy. Correlation of models for crash analysis often compares individual sensor responses to analytical predictions; a successful correlation effort is one that matched individual sensor magnitudes and times for peak values to occur. The mathematical approach evaluated in this paper decomposes observed time responses into dominant deformation shapes and their corresponding contribution to the measured response. To correlate results, orthogonality of test and analysis shapes is used as a criterion. Data from an impact test of a composite fuselage is used and compared to finite element predictions. In this example, the impact response was decomposed into multiple shapes but only two dominant shapes explained over $85 \%$ of the measured response. Test and analysis results revealed very similar deformation shapes (orthogonality values over 0.8 ). Using a vector norm criterion, maximum response values and peak time are also computed for all sensors. The norm criterion is contrasted with the commonly used single sensor criterion.
\end{abstract}




\section{List of Symbols}

$\begin{array}{cl}c & \text { Time dependent coefficients } \\ m & \text { Number of sensors } \\ n,, k & \text { Integer indices } \\ u_{i} & i^{\text {th }} \text { column of left singular vector } \\ s_{1} & \text { Diagonal matrix with nonzero singular values } \\ t & \text { Time } \\ y & \text { Time response vector } m \times 1 \\ y_{i} & \text { Time response vector for the } i^{\text {th }} \text { sensor } \\ U & \text { Left singular vector } m \times m \\ S & \text { Singular value matrix } m \times n \\ V & \text { Right singular vector } n \times n \\ T & \text { Sample time } \\ J & \text { Scalar performance index } \\ C & \text { Data matrix } m \times n \\ Z & \text { Transformed space } m \times n \\ \sigma_{i} & i^{t h} \text { singular value } \\ \text { diag } & \text { Diagonal matrix } \\ \text { MAX } & \text { Maximum value } \\ ()^{T} & \text { Transpose matrix }\end{array}$




\section{Introduction}

Finite element models and the tremendous advances in computer technologies have revolutionize they way analytical models are developed today. Dimensionality of models, a constraint in the early years of finite element modeling, is no longer a hindering factor in the development of high dimensional models to capture critical behavior during impact. With the higher dimensional models comes the need to examine all information collected from experiments to better understand and characterize the physics of impact problems. Relative and absolute motion among sensors is equally important in most correlation efforts. In the last few years, new research Refs. [1-2] has been focused on defining better metrics for use in correlation studies and to exploit the abundance of data now available but often un-used. To understand the motivation for most correlation efforts it is important to look at some important work completed in this area.

A wealth of research has been published in the impact dynamics area dealing with validation and correlation of analytical models with test. Fasanella, and co-authors in Ref. [3] discussed the analytical work conducted in preparation for the Full-scale Boeing airplane Controlled Impact Demonstration (CID). DYCAST nonlinear finite element program was used to compare fuselage crushing at two different locations and acceleration predictions at seven different locations. Whittlin and Caiafa in Ref. [4] reported on the development of a computer simulation program named $\mathrm{KRASH}$ and its application to Boeing CID. In their paper a significant amount of data was shown for model correlation against acceleration, bending moments, impact velocity, and crush distance. This comprehensive study showed data comparisons for individual sensors and uncertainty bounds for acceleration information grouped in terms of peak values. Kamat in Ref. [5] 
conducted a survey, which at the time, studied the most popular finite element analysis codes and validation efforts by comparing impact responses from a UH-1H Helicopter drop test. Data correlation for the UH-1H Helicopter had been analyzed by Whittlin and Gamon Ref. [6] using the KRASH analysis program. In this case acceleration loads at seven different locations were compared along with corresponding peak times. Also compared was the maximum deflection and permanent deformation resulting from the crash. Another excellent example of a correlation effort is that of Cronkite and Mazza in Ref. [7] comparing results from a U.S. Army's Composite Helicopter with simulation data from the KRASH analysis program. In this particular case, landing gear stroking was a key parameter compared during the correlation effort.

Impact correlation studies are conducted routinely in the automobile industry. A very interesting article by Ghandi and Hu Ref. [8] shows perhaps one of the few instances where mathematical models have been upgraded using test data to improve the analytical model for impact predictions. Un-like other work mentioned previously, this work actually uses data from test to correct errors in the model. Typical sensor data considered in the validation effort are acceleration traces at key locations in the automobile, force versus crush distance, and velocities. Another interesting article showing correlation efforts in the automobile industry is by Prasad Ref. [9]. What is interesting about this particular work is how data from front impacts is used to determine a parameter known as the coefficient of restitution. This parameter relates the approach velocity to the rebound velocity on impact. Because data is processed to determine the coefficient of restitution, analytical models from impacts can be compared based on the coefficient predictions. 
Although model correlation for aircraft and automobiles has been a very prolific area of research, no single technique has been universally accepted and used when reconciling differences between test and analysis results. Often models are compared to a small number of sensors and good correlation is claimed if a significant number of the sensors agree with tests. The approach presented here follows the work first proposed by Anderson and co-workers in Refs. [10-11]. The discussion to follow is divided into three parts; the first part presents a systematic approach to study impact data from many sensors at a time (at times hundreds of sensors) to condense information into a smaller set of key parameters for use in model correlation; the second part is discusses a technique to capture effectively relative motion information; and the third part discusses a multi-sensor performance metric for correlation of multi-dimensional finite element models. Finally, data from an impact test of a composite fuselage is used and compared to results from finite element predictions.

\section{Problem Formulation}

Impact test data can be thought to come from short duration pulses applied to a structure at one or more locations. In general the response of a linear time invariant system to a pulse can be written as an exponentially decaying time function. For linear time invariant systems, if one could match the pulse response of an analytical model with test, the model is considered to be an accurate representation of the real system. Although data from impact tests often include nonlinear behavior, the implied premise is that correlation of pulse response data with analysis is a good indicator of model fidelity. Although the premise was motivated by analysis of linear time invariant systems the procedure to be discussed next (initially proposed in Refs. 10-11) applies to nonlinear systems as well. 
Since input pulses are seldom measured during an impact test, input pulses must be inferred from carefully designed initial conditions. Using the prescribed initial conditions, the pulse response data can be grouped for analysis and correlation studies as described in the following. A pulse response data matrix with $n$ sample points sampled every $T$ seconds can be constructed as follows;

$$
Y=\left[\begin{array}{llll}
y(0) & y(T) & \cdots & y(n T)
\end{array}\right]
$$

If the are $m$ sensors, the matrix $Y$ is dimensioned $m \times n$. To examine extreme values of the multi-dimensional pulse response data, decompose the pulse history using singular value decomposition such that

$$
Y=U S V^{T}=U\left[\begin{array}{ll}
s_{1} & 0
\end{array}\right]\left[\begin{array}{l}
v_{1}^{T} \\
v_{2}^{T}
\end{array}\right]
$$

The matrix $U$ is an orthonormal matrix sized $m \times m, S$ is a block diagonal matrix $m \times n$ containing singular values, and $V$ is an orthonormal matrix sized $n \times n$. By construction the matrix $S$ can have at most $m$ nonzero singular values (for $m<n$ ) stored in the diagonal matrix $s_{1}$. Matrix partitioning of the first $m$ rows of $V$ yields $v_{1}^{T}$ and the last $n-m$ rows provides $v_{2}^{T}$. In this decomposition the matrix $U$ provides a set of basis vectors to represent the output response. In fact, any arbitrary output response can be written as

$$
y(t)=U C(t)=\left[\begin{array}{llll}
u_{1} & u_{2} & \cdots & u_{m}
\end{array}\right]\left\{\begin{array}{c}
c_{1}(t) \\
c_{2}(t) \\
\vdots \\
c_{m}(t)
\end{array}\right\}
$$

where $u_{i}$ are the columns of $U$ and $c_{i}(t)$ are time dependent coefficients. This effectively decomposes the three-dimensional motion into a set of basis vectors (static deformation shapes) and a set of scalar time varying coefficients. When looking at various impact 
scenarios, is it very informative to display the basis vectors like deformation shapes in a vibration analysis. The $c_{i}(t)$ parameters correspond to the contribution of the observed pulse in the direction of the basis vector $u_{i}$.

Pre-multiplying Eq. (3) by $U^{T}$ and using the fact that $U$ is orthonormal, yields a set of time dependent coefficients

$$
C(t)=U^{T} y(t)
$$

Equation (4) is the projection of the measured response onto the basis vectors. To examine the maximum values of the output vector, compute the inner product of the output vector and evaluate its maximum value for all times as follows

$$
J=\underset{t \in[0, n T]}{\operatorname{Max}} y^{T}(t) y(t)=\underset{t \in[0, n T]}{\operatorname{Max}} C^{T}(t) C(t)
$$

This operation simply looks at maximum value and the time for the maximum value to occur. From the singular value decomposition of the pulse matrix, the output coefficients are already evaluated by

$$
\left[\begin{array}{llll}
C(0) & C(T) & \cdots & C(n T)
\end{array}\right]=s_{1} v_{1}^{T}
$$

Substituting Eq. (6) into (5) yields

$$
J=\operatorname{Max}\left\{\operatorname{diag}\left\{v_{1} s_{1} s_{1} v_{1}^{T}\right\}\right\}
$$

From this expression, values for the maximum $C(t)$, maximum vector norm, and the time for maximum to occur are determined. It is proposed that instead of using the maximum individual sensor responses for correlation, a vector norm should be used because it is more representative of the internal deformation of the structure. The maximum vector norm 
computation is analogous to computing principal stresses and principal directions in a material.

At this point results from a finite element model and test can be compared in terms of the $U$ basis vectors, the $C(t)$ coefficients, the maximum values for $J$, and the time for the maximum values to occur. A question that one may ask is; why not compare time histories directly? There are several benefits from decomposing pulse response data using singular values. First, the basis vectors provide information on preferred directions (dominant deformation shapes in vector form) and percentage of the total response in this direction numerical value of singular values-. Second, the basis vectors are orthonormal making comparison among different models as simple as computing the inner product of vectors. Finally, shape from the output basis vectors can be examined to assess relative importance of different sensor locations.

\section{Physical Interpretation of the Singular Values}

A question that needs to be answered relates to the interpretation of singular values in terms of the data provided. Singular values have a physical interpretation with regard to the system response squared. Consider the system response in terms of the root mean square (RMS) value. Equation (1) can be used to compute the squared response as

$$
Y Y^{T}=\sum_{k=0}^{n} y(k T) y^{T}(k T)=U S^{2} U^{T}
$$

The expression to the right is a result of replacing the response matrix $Y$ with the SVD representation in Eq. (2). For the $i^{\text {th }}$ sensor the RMS response is given by;

$$
y_{i}^{r m s}=\left(\frac{1}{n} \sum_{k=0}^{n} y_{i}^{2}(k T)\right)^{1 / 2}=\left(\frac{1}{n} \sum_{j=0}^{m} u_{i j}^{2} \sigma_{j}^{2}\right)^{1 / 2}
$$


the right hand term shows explicitly the contribution from each singular value and left singular vector $u_{i j}$ to the total RMS response. Although Eq. (9) establishes a direct relation between singular values and vectors to the system RMS response, the connection is better understood by examining Eq. (8). The diagonal elements of the matrix are proportional to the square response value for each sensor point. For argument sake, let us consider a coordinate transformation such that $Z=U^{T} Y$. In this transformed output space the squared response is $Z Z^{T}=S^{2}$. Hence, if one is able to transform the output space to align it with the principal directions obtained from the SVD decomposition, the $m$ non-zero singular values squared are proportional to the system RMS response. Furthermore, the principal directions in $U$ provide a decomposition of the motion during impact arranged based on their contribution to the RMS response. It is propose that model correlation and update efforts be aimed at reconciling differences between the principal directions and singular values from test and analysis.

To demonstrate the procedure described in this section, acceleration data from an aircraft fuselage is analyzed using this technique along with analytical predictions using DYTRAN finite element program. A description of the test section and test method is described next.

\section{Description of Fuselage Section Test}

An advanced-concept, composite full-scale fuselage aircraft section with an energy absorbing sub-floor, see Figure 1, was impact tested at NASA Langley, Ref. [1]. The purpose of the test was to acquire a high quality and detailed data set for use in the test and analysis correlation studies. Extensive experience in both modeling and testing of the section has been gained. This experience has been documented in Refs. $[1,2,12,13]$. 
The fuselage section is $162.6 \mathrm{~cm}$ long with a diameter of $152.4 \mathrm{~cm}$. The design includes a very stiff floor that produces an essentially uniform global crushing of the energyabsorbing sub-floor. In the current configuration, the fuselage section contained ten 444.8 $\mathrm{N}$ lead weights; symmetrically distributed on the fuselage floor, see Figure 2 . The weights were attached to the section through bolts connected to the seat rails.

Numerous video and high-speed film cameras as well as still cameras recorded the test. In addition, data were recorded from 73 accelerometers at $10 \mathrm{kHz}$ sampling rate by an onboard digital data acquisition system. Only a sample of the floor accelerations, as indicated in Figure 2, are used in this paper. The weights have been installed to grossly approximate the point loads caused by incorporation of seats. The $444.8 \mathrm{~N}$ weight has been modeled as two $222.4 \mathrm{~N}$ concentrated weights on each seat rail in the finite element model.

The desired impact conditions were $762 \mathrm{~cm} / \mathrm{s}$ vertical velocity, no roll, pitch or yaw. The true impact conditions varied slightly from these values. As the predictions were intended to be a priori, the roll, pitch and yaw in the simulations remained at zero.

The fuselage section was densely instrumented. Correlation of the high channel count was feasible due to the streamlining of the data reduction process. In addition, the impact attitude and velocity were set to avoid catastrophic failures. In this case it was desired to crush the sub-floor without damaging the upper structure.

Before correlating with simulation results, extensive and detailed analyses of the data were performed. These analyses were intended to insure that sufficient data existed to evaluate trends. In addition, the volume of data acquired proved valuable for identifying similarities and anomalies in the results. The analyses utilized the symmetry of the test structure and desired impact condition. 
Based on the findings from the extensive data evaluations, the experimental data is considered to be of sufficiently high quality to adequately evaluate, as well as to guide the development of the correlation methodologies.

\section{Description of finite element model}

The finite element model is shown in Figure 3. The model is comprised of approximately 30,000 elements and 30,000 nodes. The stiff structural floor was modeled as two laminated composite face sheets with a foam core. The foam core is represented using solid elements assigned linear elastic material properties. The composite face sheets are represented with linear elastic orthotropic material properties. The upper section is also modeled with a foam core with laminated composite orthotropic face sheets. The sub-floor section has solid elements with orthotropic face sheets on the interior surfaces. The accuracy of the crash simulations for this model is directly dependent on the accuracy of the sub-floor foam material properties. A stress-strain table was supplied for the FOAM2 material properties in the model. Additional details regarding the modeling approach are found in Ref. [12].

\section{Composite Fuselage Correlation Results}

To demonstrate the correlation approach using a realistic example, data from a drop test of the composite fuselage shown in Fig. 1 was used and compared to results using DYTRAN finite element simulation program. As described before, accelerometer data was collected from many locations but only a subset was used in this example problem. In particular, the subset of 19 accelerometers mounted on the lumped masses grid shown in figure 2 was selected for analysis. Acceleration time histories from channels 9-12 are shown in figure 4. Time for the acceleration values to reach their maximum (in the vector norm sense) was evaluated according to Eq. (7) to be 0.031 seconds for test and 0.028 
seconds for analysis. A vertical line at 0.031 second is shown to indicate the time when the maximum occurred. Note that the time for the maximum vector norm to occur is not and it needs not to coincide with the time when peak acceleration values are observed. This is an important distinction when examining individual sensors versus a group of sensors. The output root mean square values (RMS) were computed for each sensor and a comparison between test and analysis is shown in figure 5. Analysis RMS values for most sensor outputs are within $10 \%$ of predictions. Output RMS comparisons helps analyst identify sensors improperly filtered and/or faulty sensors.

Time histories data from the grid of accelerometers placed on lead masses, as shown in Fig. 2, were fed into the singular value decomposition algorithm. Basis vectors computed for the accelerometer grid provides a spatial description of the relative motion among the various sensors in the z-direction. Gravity is also in z-direction. Using the accelerometer grid geometry and the basis vectors computed from test and analysis, figure 6 shows a display of the two dominant basis vectors; test vectors at the top (6a-b) and analysis vectors at the bottom (6c-d). Since the vectors are normalized, one needs to be concern with is the overall shape and not with the absolute magnitude. To examine similarities between two vectors, the inner product is often used. All computed test basis vectors inner products against analysis are shown in figure 7. The ordinate corresponds to test basis vector number, the abscissa corresponds to analysis basis vectors, and the colorbar indicates the absolute value of the inner product (dark colors indicate values near 1). Note the first two basis vectors (lower left corner) correlated well (orthogonality over 0.9 ). There are several other basis vectors with orthogonality values worth investigating. For example, test basis vector 4,8 and 17 paired well with analysis basis 3,11 , and 19 , respectively. The percent 
contribution from each basis vector to the total response is shown in figure 8 as a function of the basis vector number. When examining the percent contribution to the observed acceleration response, the first two bases vector from analysis contributed $86.3 \%$ whereas the first two test bases contributed $62.7 \%$. For the test basis vectors 4,8 and 17 , the orthogonality values were higher than 0.69 , but their contribution to the response ranged from $8.4 \%$ to $0.5 \%$. Figure 9 shows the maximum acceleration distribution at peak time for all accelerometers; test (red) and analysis (blue). Acceleration distribution at peak time compared well at $y=0$ but it gets worse as $\mathrm{y}$ increases, with test showing lower values than analysis.

A few final comments about the results are in order at this time. First, the time computed for the peak vector norm to occur is different from the peak time computed for individual sensors. Second, with this approach there is a systematic way to analyze, decompose, and display results from multiple sensors during an impact test that takes into account spatial and temporal relationships among the different sensors. In fact, three-dimensional motion is easily reconstructed from the basis vector information, if all the sensors used are of the same type. Note that mixing different sensor types would destroy the significance of the spatial relationship in the basis vectors. To avoid this problem, sensors of the same type should be grouped and analyzed together. Third, the basis vectors can be used for pre-test selection of sensor locations to ensure data collection in areas were significant changes occur during impact. In the composite fuselage example, fewer sensors would have been sufficient to reconstruct the motion on impact but with more complicated geometries this may not be the case. Finally, the issue on how to use the basis vectors to guide model updates needs to be addressed. However, it is clear that improvements in the model would 
need to be directed towards reconciling test and analysis basis vectors. In this particular case, changes to the model need to concentrate in the areas where the test basis vector number 3 differs from analysis.

\section{Concluding Remarks}

A mathematical formulation using singular value decomposition has been presented to analyze impact data from tests and finite element simulations. In terms of this new formulation, correlation of models with test is based on comparing two critical features; basis vectors (extracted from time histories using SVD) and time history participation factors. Once the basis vector are computed from test and analysis time histories orthogonality of the basis vectors provides a clear quantifiable indication of similarities and differences between the analytical model and test. The idea is similar to that of correlating mode shapes in a vibration experiment. Using time history participation factors for each basis vector one can use the differences in the dominant basis vectors to pinpoint areas in the model where update efforts need to be concentrated. In addition, the procedure facilitates identification of bad sensors and is ideal for sensor placement guidance.

In order to validate the mathematical procedure, impact data from analysis and test of a composite fuselage was analyzed. In this example two dominant basis vectors (out of twenty) were identified that accounted for over $62 \%$ of the response during test and $86 \%$ for the analysis. Percent contribution to the total response is based on decomposing the time histories into basis vectors that when combined are able to reconstruct the measured time history exactly. Individual sensors RMS calculation showed that the analytical model for the most part over-predicted the system response by $10 \%$. Computed orthogonality values of over 0.9 for the two dominant basis vectors verified the similarities between test 
and the analytical model. In addition, the spatial relationship among multiple accelerometers at the time when the maximum vector norm acceleration occurred is consistent experimental observations. Another parameter often considered in correlation studies is the time for peak response values to occur. This calculation is normally done one sensor at a time or using one critical sensor. In this new approach a vector norm using all sensors is used instead and the time for the maximum vector norm to occur is recorded. The computed time for the peak norm vector to occur for test and analysis was within 3 msec. Overall, the finite element model compare very well with predictions.

\section{References}

1. Bark, L. W.; and Lyle, K. H.: Progress toward Understanding Correlation of Crash Test with Crash Simulation. Proceedings of the Third International KRASH Users' Seminar, Tempe, AZ, January 8-10, 2001.

2. Lyle, K. H.; Bark, L. W.; and Jackson, K. E.: Evaluation of Test/Analysis Correlation Methods for Crash Applications. Proceedings of AHS $57^{\text {th }}$ Annual Forum, Washington, D. C., May 9-11, 2001.

3. Fasanella, E.L., Hayduk, R.J., Robinson, M.P., Widmayer, E.; “Analysis of a transport Fuselage Section Drop Test,” Research in Structural Dynamics, 1984, Pg. 347-368.

4. Whittlin, G., and Caiafa, C.A.; "Transport Airplane Crash Simulation, Validation, and Application to Crash Design Criteria." AGARD, Energy Absorption of Aircraft Structures as an Aspect of Crashworthiness. AGARD-CP-443, 1988.

5. Kamat M.P.; "Survey of Computer Programs for Prediction of Crash Response and of Its Experimental Validation," Measurement and Prediction of Structural and 
Biodynamic Crash-Impact Response. Proceedings of the Winter Annual Meeting of the ASME, Dec. 1976, Pg 33-48.

6. Whittlin, G. and Gamon, M.A.; "Experimentally Verified Analytical Techniques for Predicting Vehicle Crash Responses," Proceedings of the AIAA $11^{\text {th }}$ Annual Meeting and Technical Display, AIAA Paper No. 75-273, Feb. 1975.

7. Cronkhite, J.D., and Mazza, L.T.; "KRASH Analysis Correlation with the Bell ACAP Full-Scale Aircraft Crash Test," Proceedings of the National Technical Specialists' Meeting on Advanced Rotorcraft Structures: Requirements Vs. Opportunities, a Look at the Future. VA, Oct. 1988.

8. Gandhi, U.N., and Hu, S.J.; "Data-Based Approach in Modeling Automobile Crash," International Journal of Impact Engineering, Vol. 16, No. 1, Feb. 1995.

9. Prasad, A.K.; "Coefficient of Restitution of Vehicles Structures and Its Use in Estimating the Total $\Delta \mathrm{V}$ in Automobile Collisions," AMD- Vol. 126/BED-Vol. 19 Crashworthiness and Occupant Protection in transportation Systems, ASME, 1991, Pg. 217-246.

10. Anderson, M.C.; Gan, W.; and Haselman, T.K.; "Statistical Analysis of Modeling Uncertainty and Predictive Accuracy for Nonlinear Finite Element Models," Proceedings of the $69^{\text {th }}$ Shock and Vibration Symposium, Minneapolis/St. Paul, MN, 1998.

11. Anderson, M.C.; Haselman, T.K.; and Crawford, J.E.; "A Toolbox for Validation of Nonlinear Finite Element Models," Proceedings of the $6^{\text {th }}$ LS-Dyna User's Conference, Dearborn, MI, May 2000. 
12. Jackson, K. E.; Fasanella, E. L.: Development of a Crashworthy Composite Fuselage Concept. Proceedings of the 2000 Advances in Aviation Safety Conference (P-355), Daytona Beach, FL, April 11-13, 2000.

13. Jackson, K. E.; Fasanella, E. L.; and Kellas, S.: Development of a Scale Model Composite Fuselage Concept for Improved Crashworthiness. Journal of Aircraft, Volume 38, No. 1, January-February 2000, p. 95-103. 


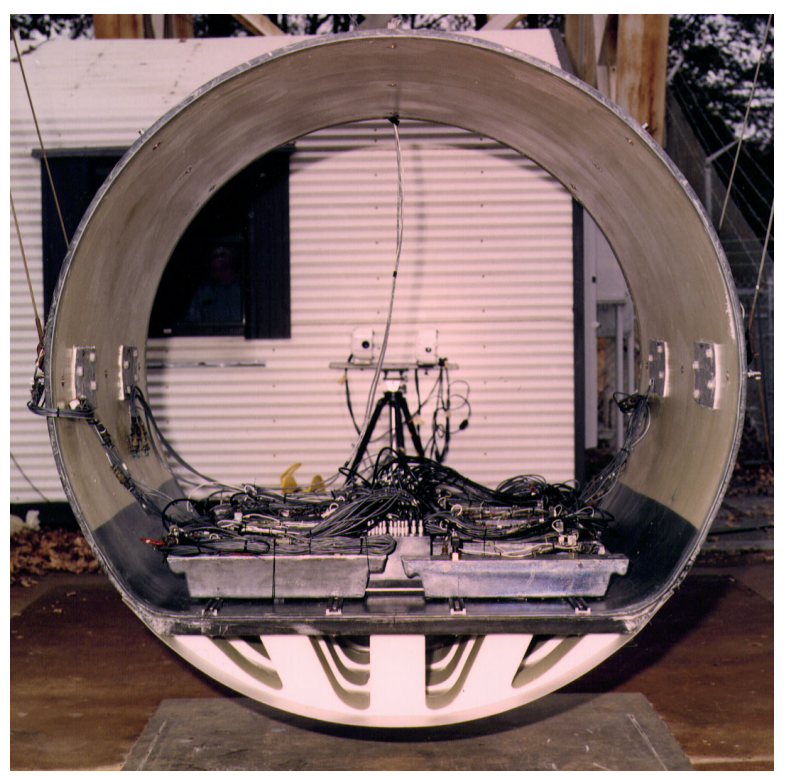

Figure 1. Photograph of fuselage section.

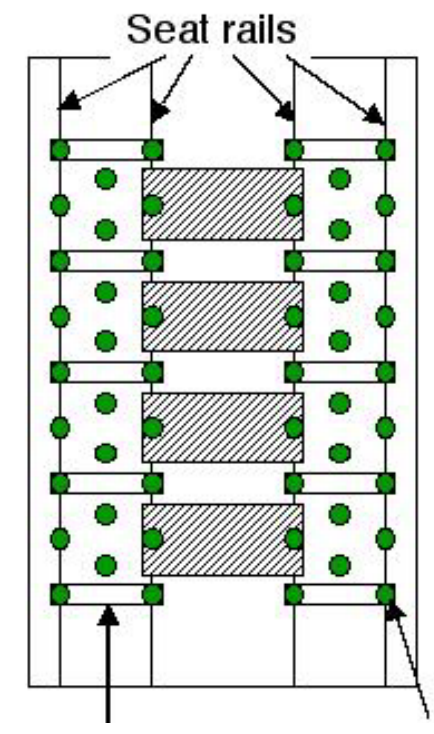

444.8 N lead Masses

Accel.

Figure 2. Schematic of instrumentation placed on the floor.

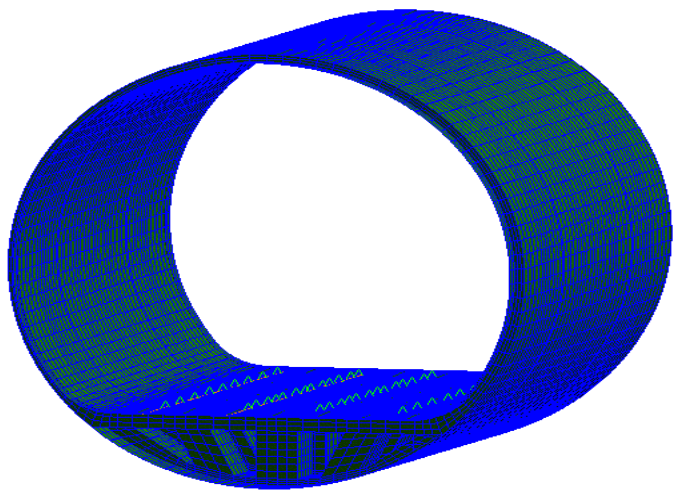

Figure 3. Finite element model of composite fuselage 

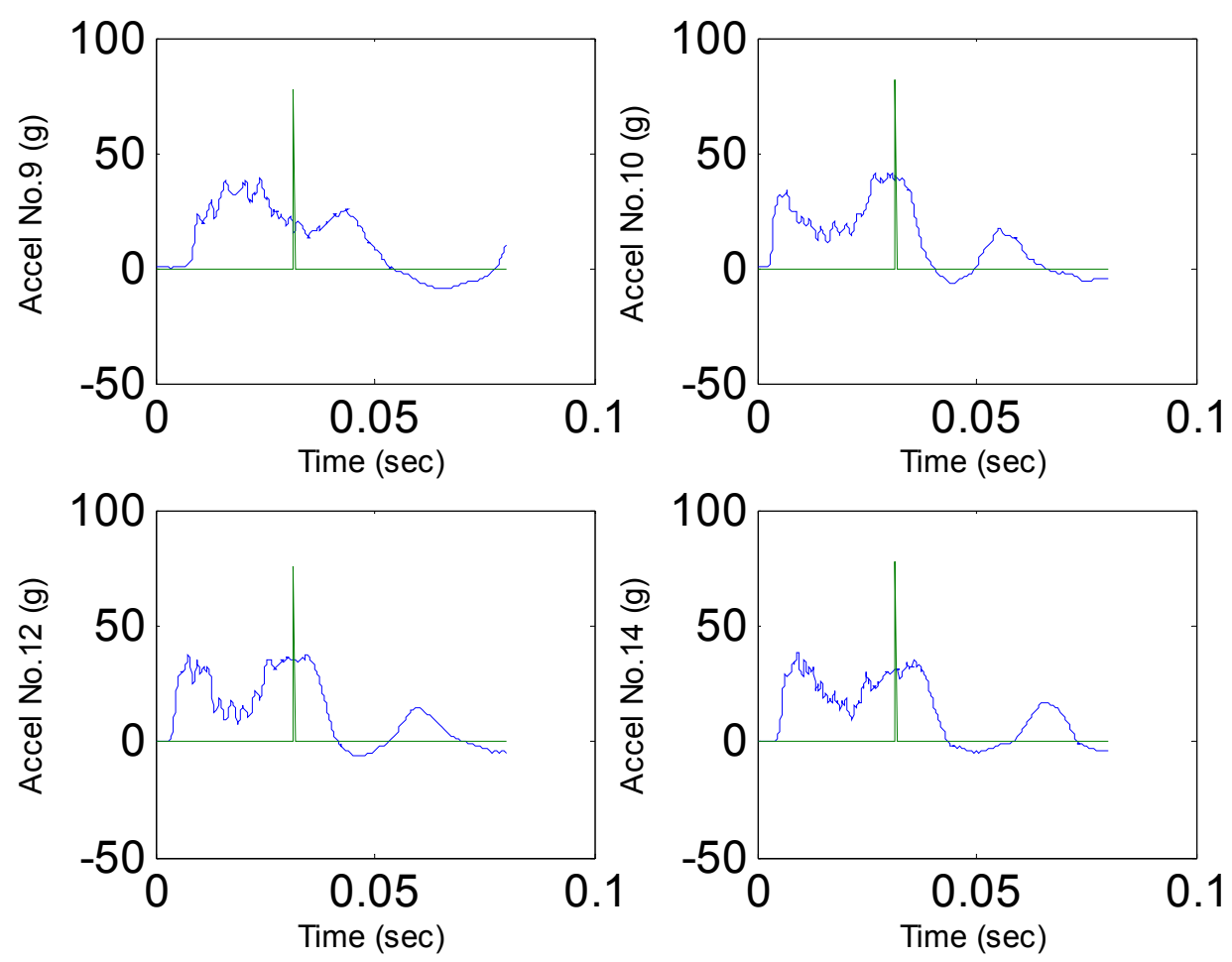

Figure 4. Typical acceleration time histories for channels 9,10,12 and 14. Marker indicates the time for maximum output vector norm.

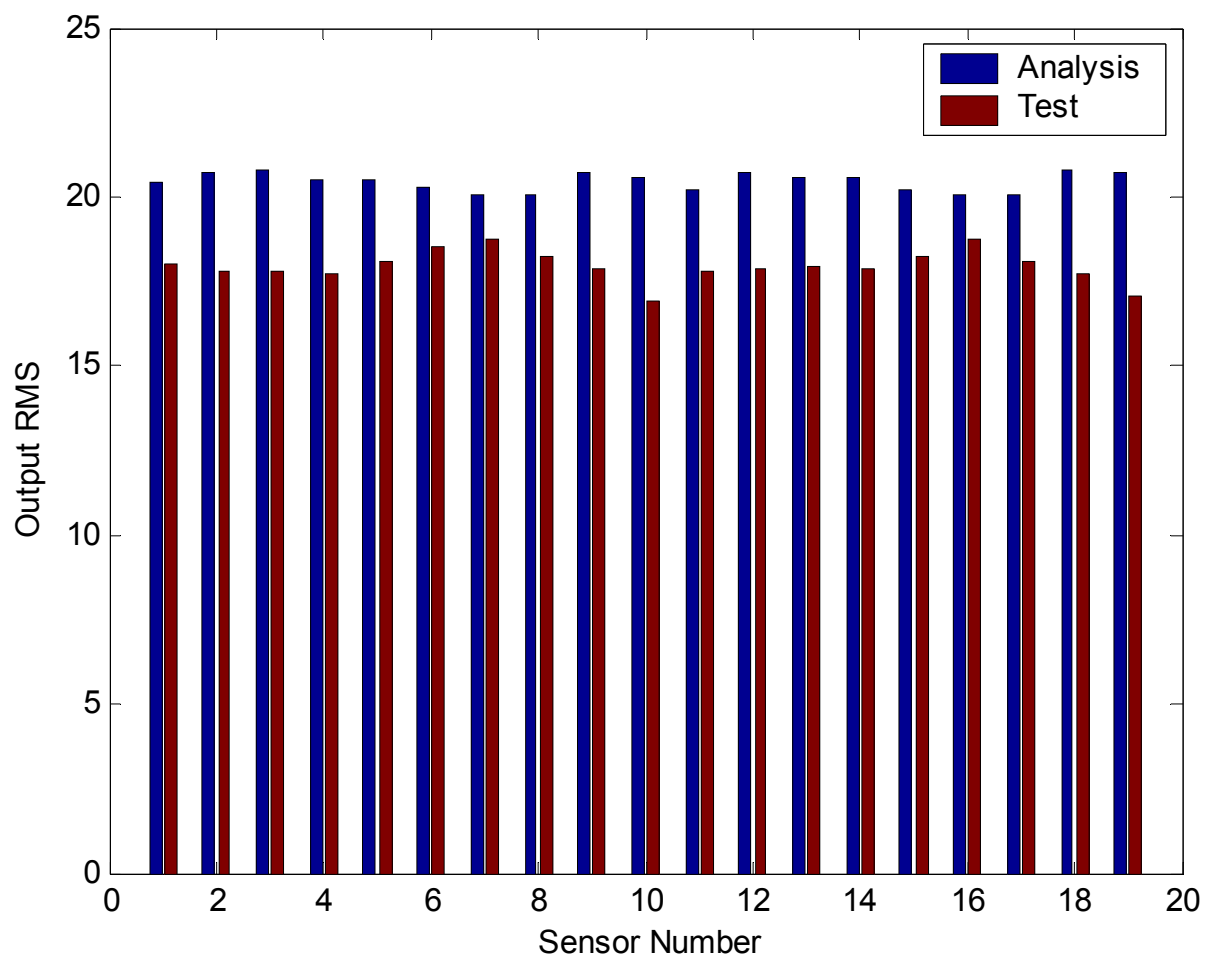

Figure 5. Comparison of test and analysis output RMS values. 


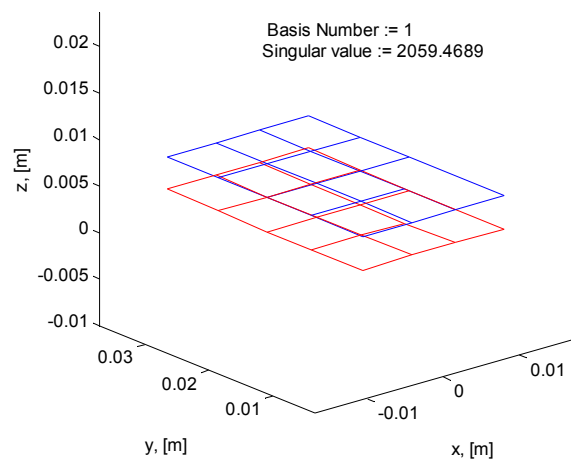

a) Test basis vector No. 1

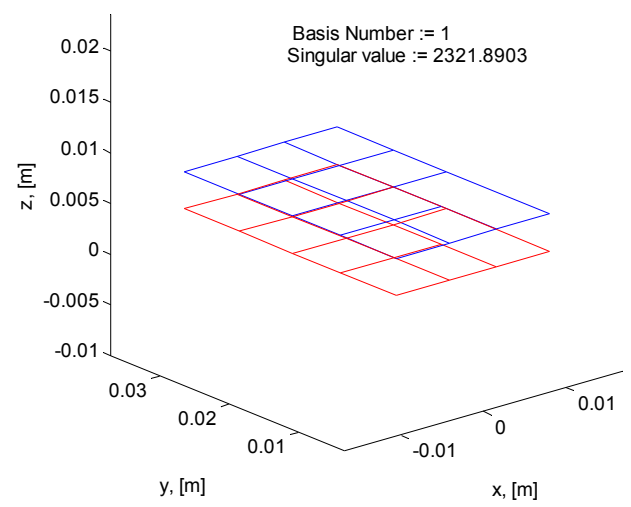

c) Analysis basis vector No. 1

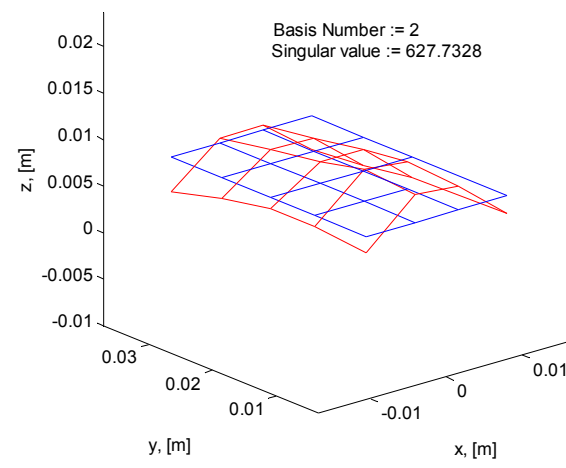

b) Test basis vector No. 2

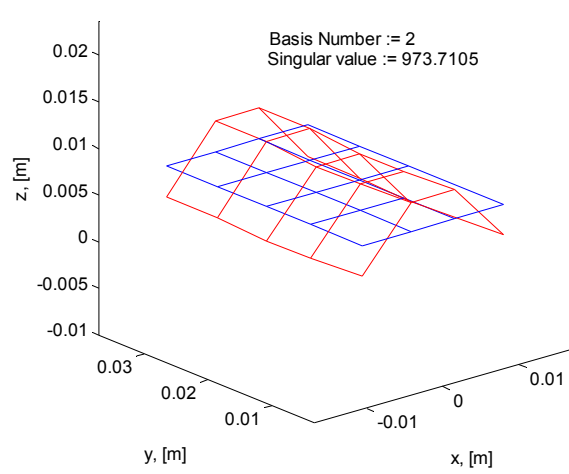

d) Analysis basis vector No. 2

Figure 6. Comparison of computed basis vectors for test and analysis

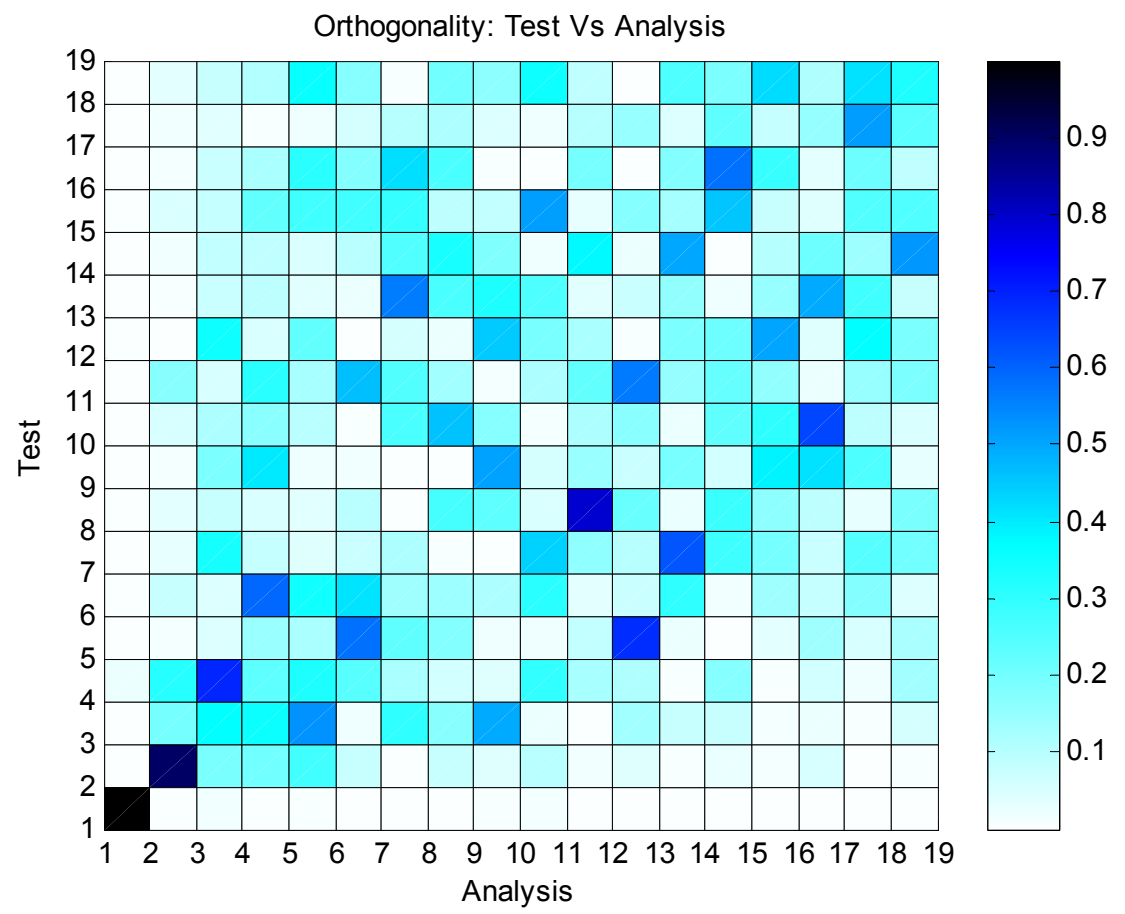

Figure 7. Orthogonality of test versus analysis basis vectors 


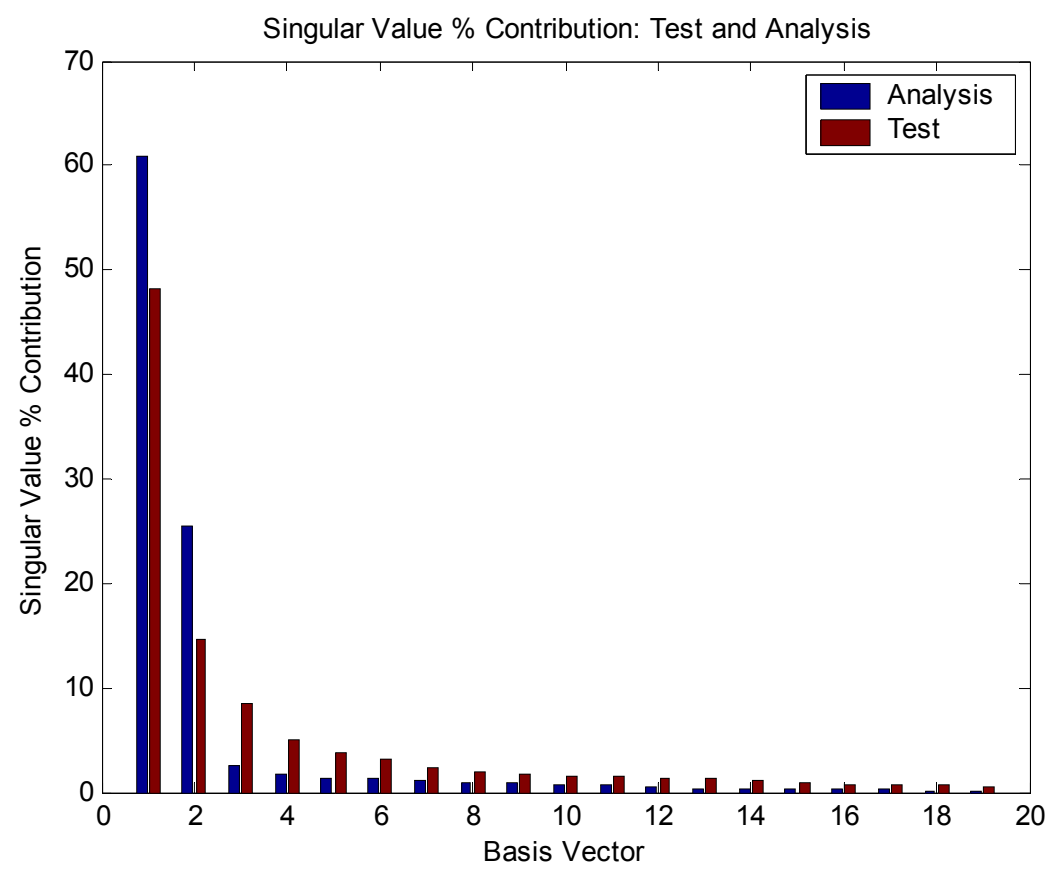

Figure 8. Comparison of singular value \% contribution for test and analysis

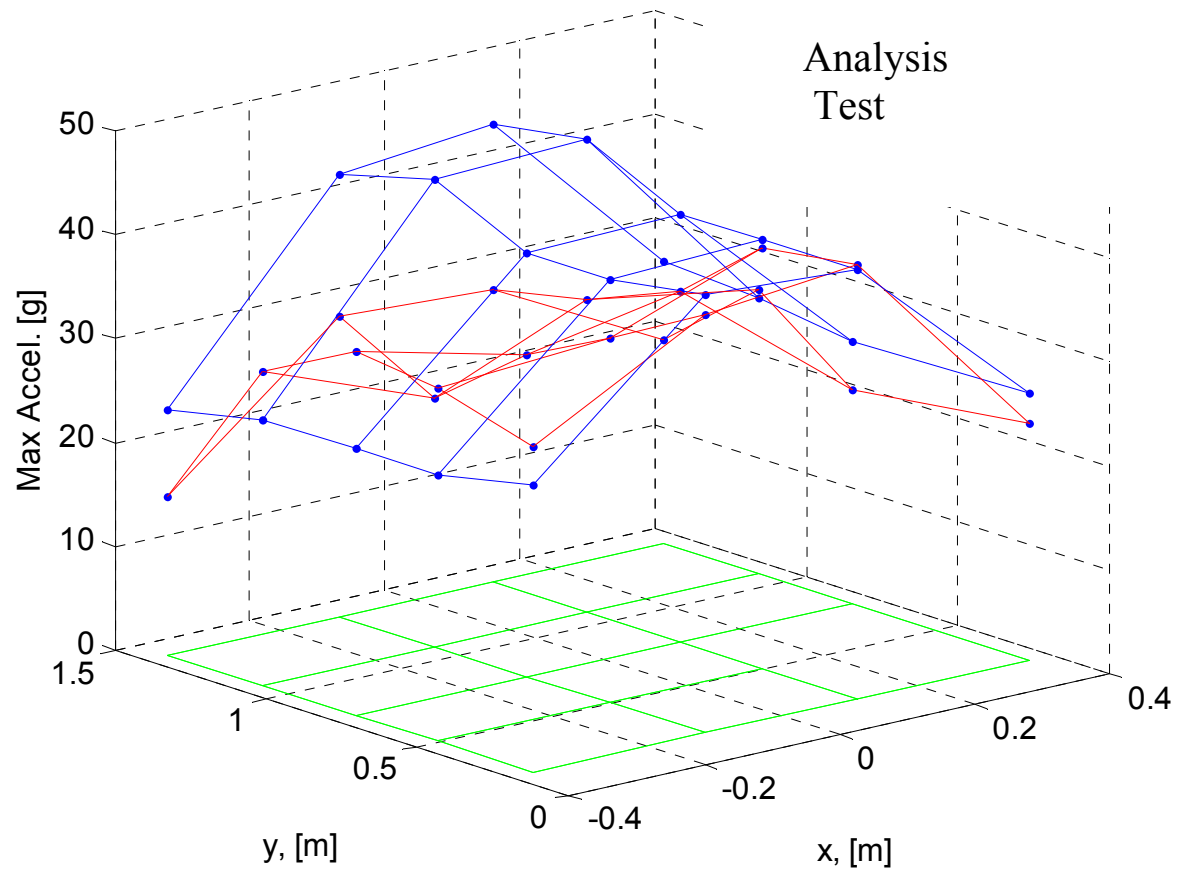

Figure 9. Comparison of maximum floor acceleration at the time when the computed output vector norm is maximum. 


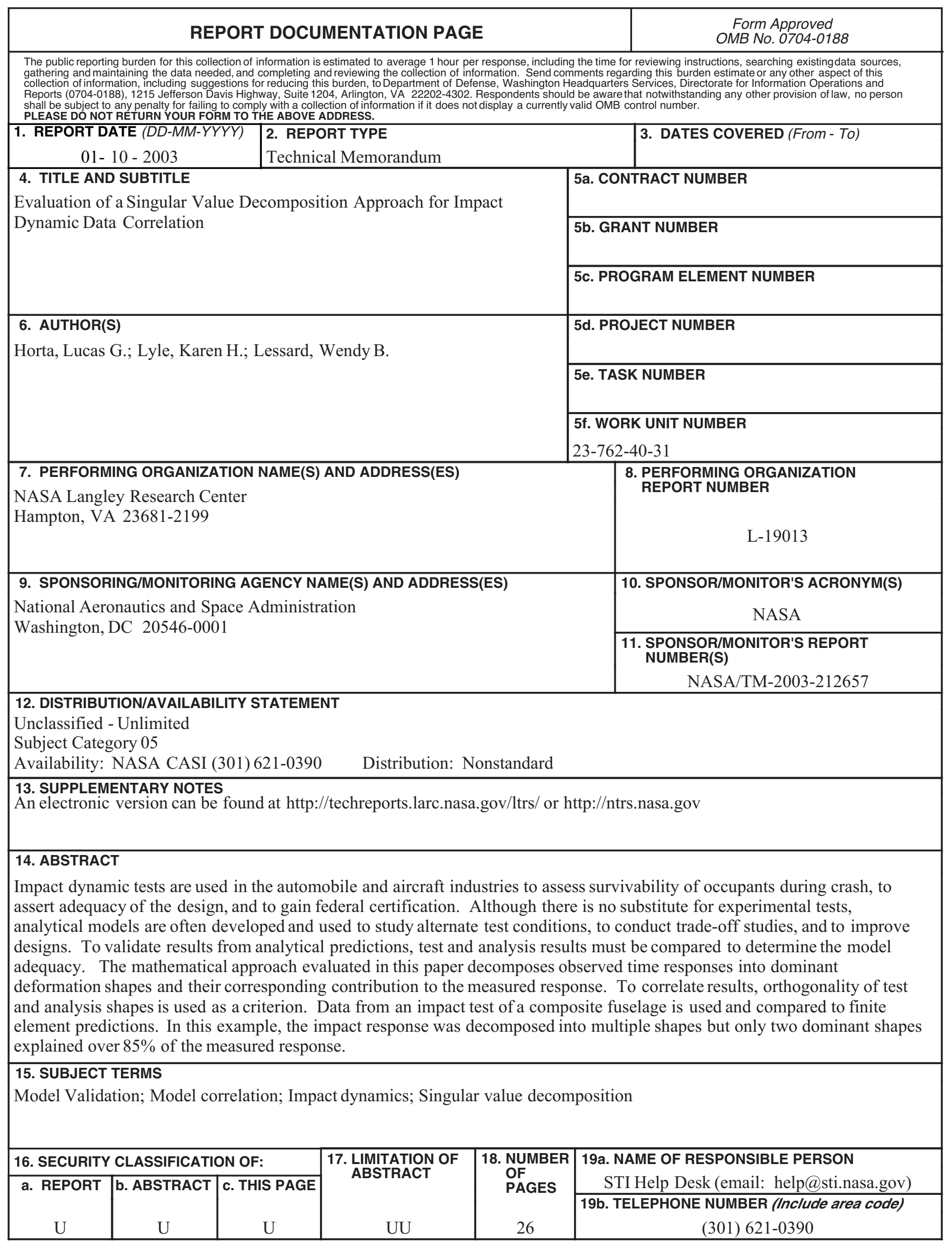

